\title{
RETROSPECTIVE COHORT STUDY WITH SURVEY ANALYSIS OF BREAST CANCER SUBMITTED RADIOSURGERY BY CEREBRAL METASTASIS IN 10 YEARS
}

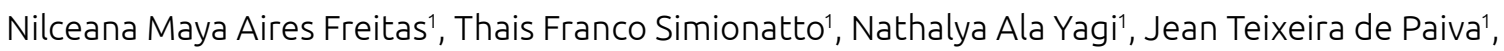
Thais de Toledo Lima Santana', Sebastião Berquó Peleja', Mattheus Humberto do Vale'1, Silvia Sousa Pires ${ }^{1}$

${ }^{1}$ Hospital Araújo Jorge - Goiânia (GO), Brazil.

Objectives: Breast cancer is the second most common type of cancer associated with brain metastasis. Symptomatic brain metastasis are diagnosed up to $16 \%$ of patients with metastatic breast cancer. The risk of brain metastasis in ten years depends on the phenotypic subtypes of breast cancer. Luminal A with a risk of 0.7\%; Luminal B, 12\%; Luminal HER2 Positive, 8\%; HER2, 12\%; and Triple Negative, 7\%. Stereotactic Radiosurgery (SRS) is one therapeutic modalities used to treat of brain metastases, which offers local control and survival comparable to neurosurgery. This study aims to evaluate the epidemiological profile of patients submitted to radiosurgery, correlate survival, and time tumor progression in the brain. Methods: Data were collected from patients with diagnosis of breast cancer brain metastasis treated with SRS from 2007 to 2017 at Hospital Araújo Jorge/Goiânia/Brazil. It was analyzed immunohistochemistry pattern, number of lesions treated with SRS, neurosurgery, whole brain radiotherapy (WBRT). Statistical analysis was performed using Fisher's test and Kaplan-Meier curve, considering 5 criteria separately: overall survival (OS), progression-free survival (PFS) for the central nervous system (CNS) after radiosurgery, brain disease free survival (DFS) after diagnose and survival after radiosurgery for brain metastasis (SRC). Results: In a time 10 years, total 78 patients with brain metastasis underwent to SRS, 71 (91\%) were treated with a single dose procedure and six (8\%) with a five fractions procedure. Among these, 59 (76\%) were submitted to SRS in a single, $15(19 \%)$ in a double, and four (5\%) in three procedure. The number of lesions treated ranged from one to six metastases. Most patients were triple negative (30\%) and HER2 positive (30\%). Twenty patients (26\%) underwent to WBRT, 60\% after SRS. 34 patients (44\%) presented brain disease progression after the first SRS, in the mean time of 9 months and 17 patients (22\%) died after 19 months, and OS 78\% The mean of follow-up was 12 months, with the maximum of 60 months. Conclusion: SRS is a therapeutic tool that improves survival, providing better quality of life and lower neurocognitive deficit when compared with WBRT in the treatment of brain metastases. WBRT can be avoided up to 3/4 of the population that was treated by SRS as a primary treatment for breast câncer brains metastasis and more than a half presented with stable disease control at CNS after radiosurgery. 